Perspectiva de Familia (Perspect. Fam.) ISSN 2415-5187 (impreso), ISSN 2519-1748 (en línea), año 2019, vol. 4, pp. 97-126

\title{
Perú: Influencia del enfoque de género en las políticas públicas familiares
}

Peru: Influence of the gender approach in family public policies

Erika J. Valdivieso

Universidad Católica Santo Toribio de Mogrovejo, Perú

Correspondencia: evaldivieso@usat.edu.pe

Recibido: 14-08-2019. Revisado: 14-10-2019. Aceptado: 31-10-2019

Citarlo como: Valdivieso, E. (2019). Perú: Influencia del enfoque de género en las políticas públicas familiares. Perspectiva de familia, 4, pp. 97-126.

\section{Resumen}

En los últimos años, el enfoque de género ha sido en el Perú —como en otros países latinoamericanos - la herramienta principal para el desarrollo de políticas públicas relacionadas con la familia. Este enfoque considera que los roles y tareas de mujeres y hombres, así como su sexualidad, son construcciones culturales basadas en situaciones de asimetría y relaciones de poder. Cuando se repasan las políticas públicas relacionadas con la familia, se evidencia que no se logran los resultados esperados pese a que en ellas se ha incorporado el enfoque de género. Al analizar las causas es posible identificarlas con la contradicción entre los postulados de la teoría de género y lo que representa la familia para la sociedad.

Palabras clave: Políticas públicas, enfoque de género, políticas familiares, familia.

\begin{abstract}
In recent years, the gender focus has been in Peru —as in other Latin American countries - the main tool for the development of public policies related to the family. This approach considers that the roles and tasks of women and men, as well as their sexuality, are cultural constructions based on situations of asymmetry and
\end{abstract}


power relations. When reviewing public policies related to the family, it is evident that the expected results are not achieved although the gender approach has been incorporated into them. By analyzing the causes it is possible to identify them with the contradiction between the postulates of the theory of gender and what the family represents for society.

Key words: Public policies, gender approach, family policies, family.

\section{Punto de partida}

Cuando nos referimos a la familia, debemos partir de un hecho ineludible: la familia es indispensable para el hombre y para la sociedad; podría decirse que es coexistencial al hombre, y que, como señala D'Agostino (2002):

[...] es un principio antropológico [que]; no es reducible ni siquiera a un mero dato de consanguineidad biológica, ni siquiera a una dimensión histórica-cultural, sino pertenece a la estructura constitutiva del ser humano. Es en la familia y a través de la familia que el hombre adquiere, instaura y porta a cumplimiento la propia identidad personal y más genuinamente humana, que es identidad relacional. (p. 267)

En otras palabras, la familia es una institución natural.

En contraposición, surge la postura planteada por el feminismo de género que critica la «imagen fantasmal de una familia ideal» e insiste en que esta es una institución articulada a la sociedad, con una dinámica interna en la cual se reproducen relaciones de poder (Puyana, 2007). Considera que la familia es el espacio donde históricamente la mujer ha sido subordinada por el patriarcado y una forma de enriquecimiento de los varones a costa de la mujer y los hijos. Por ello, «las instituciones sociales [como la familia] son construcciones culturales susceptibles de ser transformadas por efecto de los cambios en las relaciones sociales» (Montaño, 2005, p. 97).

Más allá de la discusión teórica que ambas posturas plantean, cabe preguntarnos si el enfoque con el que se asumen las políticas públicas dirigidas a la familia influye en el desarrollo y efectividad de las mismas para el cumplimiento de sus objetivos. Actualmente, en el marco de las políticas públicas, el enfoque de género destaca entre todos los implementados por el Estado peruano. El objetivo de este es -entre otros- visibilizar a la mujer y lograr su empoderamiento en un plano de igualdad con el varón. 
Ahora bien, pese a los esfuerzos del Estado en la implementación del enfoque de género, la percepción -en lo que concierne a políticas públicas relacionadas con la familia - es que no se ha logrado la igualdad deseada entre hombres y mujeres, no se han reducido los índices de violencia contra la mujer, no se ha reducido el embarazo adolescente, no hay indicadores positivos de fortalecimiento de la parentalidad, la corresponsabilidad o el matrimonio. Por ello, se hace necesario analizar la influencia de la aplicación de este enfoque en las políticas públicas no solo por su - cada vez mayor - cuestionamiento a nivel político y social, sino porque los problemas que se pretenden solucionar tienen que ver directamente con la concepción del individuo, el matrimonio y la familia que, como sabemos, se constituye como el elemento fundamental para el desarrollo de la sociedad.

Analizaremos a lo largo de este trabajo si los postulados básicos del enfoque de género son apropiados para desarrollar políticas públicas dirigidas a la familia de manera eficiente, o si existe una contradicción que hace incompatible la aplicación del enfoque de género con las políticas públicas familiares. Para esto, se realizará un breve análisis de las recientes normas con enfoque de género que tienen relación con la familia.

\section{Delimitación conceptual del Enfoque de género}

Es un hecho que en la historia de la cultura occidental, el rol de la mujer y la relación entre hombres y mujeres han sufrido una notable evolución. También podemos afirmar que las funciones asumidas y las relaciones entre los sexos tienen especial vinculación con la familia. La prevalencia de la autoridad masculina y la postergación de lo femenino en la sociedad y la familia, hasta el siglo XIX, aproximadamente, se basaba, como señalan Gil y Vallejo (2013), en una concepción antropológica y cultural que consideraba al hombre como un ser superior a la mujer, básicamente por cuestiones físicas. La consecuencia es que la mujer tenía menos oportunidades en el ámbito de la educación y el trabajo, en la política o en la vida social en general.

Elósegui (2005) sostiene que la relación de «dependencia de la mujer con respecto al varón iba unida a dos presupuestos: exaltación de las diferencias, negando la igualdad, y una identificación entre sexo biológico y funciones sociales, hoy denominadas funciones de género» (p. 126). Y en esa distribución de roles, para la mujer estaba reservado el ámbito de lo privado (la administración del hogar y la responsabilidad por el cuidado y educación de los hijos) y el varón asumía las funciones relacionadas con lo público (el trabajo fuera de casa, la manutención del hogar, el ámbito de las decisiones y participación política, etcétera). 
El enfoque de género pretende entonces ser el instrumento para lograr la plena igualdad entre hombres y mujeres a partir de la equiparación de sus roles en la sociedad. Sin embargo, pese a que en lo superficial podría considerarse una aspiración legítima -y por ello, se explica el apoyo de la mayoría-, cuando se profundiza en los fundamentos teóricos que lo originan, se puede advertir alguna distorsión en su causa.

Cuando intentamos desarrollar un concepto del término género, nos encontramos con dificultades, debido a que es una noción desarrollada en dos planos: el psicológico-médico y el político. Ambos parten de una particular forma de interpretar la relación entre el sexo (varón-mujer) y los roles que desempeña cada uno en la sociedad (género).

Desde el planteamiento biológico (psicológico-médico), fueron Stoller y Money (Aparisi, 2009) quienes precisaron la distinción radical entre sexo (relacionado con la biología, hormonas, genes, morfología, etc.) y género (que se relaciona con la cultura, psicología, sociología), de tal manera que este último sería lo social o culturalmente construido, y el sexo, lo biológicamente determinado. Marcan, así, una independencia total entre el sexo y el género, el mismo que puede construirse — como el lenguaje- por patrones socioculturales inducidos.

Por otro lado, desde el plano político, la teoría del género se encuentra ligada «a una concepción socialista que ponía por delante el análisis de la dominación social, a la diferencia sexual» (Aguilar, 2008, p. 21). Desde aquí se plantea que estas diferencias están marcadas por una relación desigual de poder entre mujeres y varones. El género es entonces una construcción social de lo femenino, y se construye bajo el patriarcado dominante que la mujer sufre en la familia. En esta concepción, el patriarcado se define como:

La manifestación de institucionalización del dominio masculino sobre las mujeres y los niños de la familia, dominio que se extiende a la sociedad en general. Implica que los varones tienen poder en todas las instituciones importantes de la sociedad y que se priva a las mujeres de acceso de las mismas. Las mujeres no tienen aquí, ningún tipo de poder, ni de derechos, influencias o recursos. (Facio \& Fries, 1999, p. 45) 


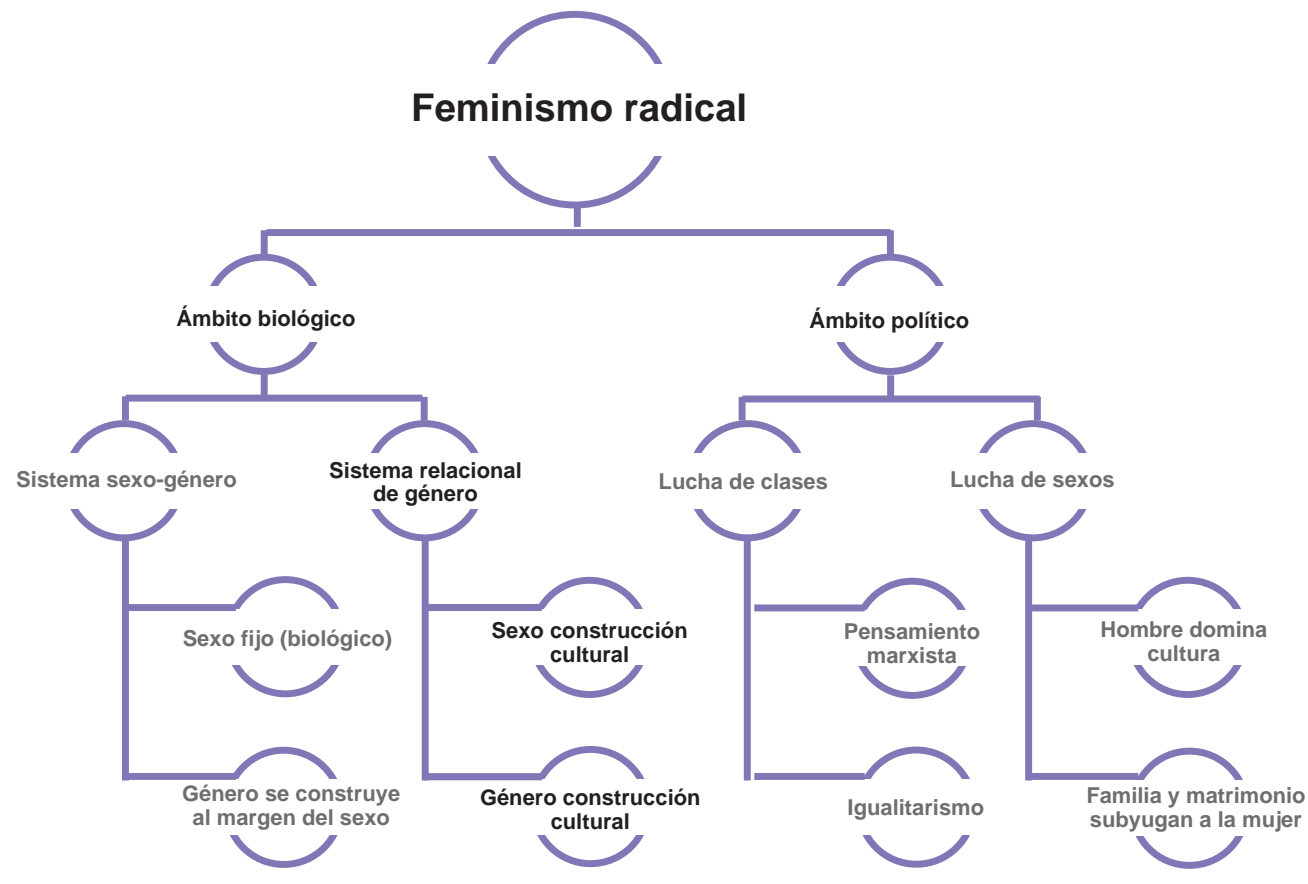

Figura 1.

\section{Desarrollo del pensamiento feminista radical}

\section{Elaboración propia}

\section{La idea de género en los postulados feministas}

Desde la perspectiva histórica, el término género empieza a utilizarse desde el llamado feminismo radical (a partir de los años 6o). Antes, el feminismo sufragista (S. XIX-1940) planteó la reivindicación de la igualdad de derechos entre hombres y mujeres, materializados, entre otros, por el derecho al voto y el aumento de la presencia de la mujer en los ámbitos políticos y académicos, sin atribuir una responsabilidad específica en la generación de la desigualdad. Sin embargo, como señala Aparisi (2002), «de acuerdo con sus presupuestos ideológicos, conllevó una nueva visión del lugar de la mujer en la sociedad, mucho más individualista» (p. 9), alejada de la maternidad y la vida familiar, y se llega a entender que el ámbito de lo privado, de la familia, supone una seria desventaja para la mujer en términos de ocupar un lugar en el espacio público. Esta se empieza a situar ante un dilema que enfrenta la familia (y su vocación de esposa y madre) y lo social, que no es otra cosa que el inicio del abandono de la feminidad. 


\section{Feminismo radical (1960-1980)}

Es en esta época cuando empieza a tomar fuerza la teoría de género. El planteamiento inicial del concepto de género (con los estudios de Stoller, de 1968) (Aparisi, 2009) implica que lo determinante en la identidad sexual no es el sexo biológico, sino el hecho de ser socializado desde el nacimiento o antes como perteneciente a uno u otro sexo. Luego, es Ann Oakley que, en 1972, incluye el término género en las ciencias sociales (Stolke, 2004). A partir de entonces, la distinción entre sexo y género «fue usada por el feminismo para explicar la subordinación de las mujeres como algo construido socialmente y no justificada en la biología» (Facio \& Fries, 1999, p. 32). Ahora, si bien se entiende que el género es fluido, histórico y cambiante, el sexo mantiene su carácter de «lo dado», permanente o inmutable (aunque no necesariamente condicionante del género). Este es el denominado sistema sexo-género.

Para el sistema sexo-género, el término "género alude tanto al conjunto de características y comportamientos como a los roles, funciones y valoraciones impuestas dicotómicamente a cada sexo a través de procesos de socialización, mantenidos y reforzados por la ideología e instituciones patriarcales» (Facio \& Fries, 1999, p. 34). Así, las mujeres tienen desventaja, pues se les reserva las tareas referidas al ámbito del hogar, la maternidad y el cuidado de los hijos, lo que claramente supone una limitación para los objetivos de plena participación pública.

Entre sus representantes están Kate Millet, Shulamit Firestone, Monique Witting, Germaine Greer, Adrienne Rich, el Movimiento Mujeres Radicales (conocido por la elaboración del Manifiesto Scum, de 1967), entre otras. Pese a que se trata de los primeros planteamientos sobre la teoría de género, cabe hacer una diferencia entre dos corrientes del feminismo (desde la concepción política) que se manifiestan en esa época: aquella que atribuía al sistema de propiedad la responsabilidad de la subyugación femenina (lucha de clases, corriente marxista), y el movimiento feminista radical, que, al contrario, entendía que era el hombre y las instituciones como el matrimonio y la familia quienes promovían esta subyugación (lucha de sexos).

La corriente marxista relaciona el patriarcado con el surgimiento de la propiedad privada y el capitalismo (con base en Engels y Marx), por lo que sostiene que únicamente con la revolución socialista se puede lograr la liberación femenina y de las clases oprimidas (en las que, además de las mujeres, se incluyen minorías sexuales, étnicas). Esto explica que quienes, siendo feministas, buscan lograr la protección laboral de las mujeres embarazadas, por ejemplo.

Por otro lado, el llamado feminismo radical sostiene que la igualdad para las mujeres se logra por la eliminación de cualquier diferencia entre los sexos y la decons- 
trucción de lo que se entiende por femenino o masculino, que - al no depender de lo biológico- es producto de una construcción cultural que ha mantenido históricamente sometida a las mujeres. En este sentido, si bien pueden llegar a admitirse las diferencias sexuales, este dato biológico o natural se considera absolutamente irrelevante para la construcción de una identidad sexual.

Ambos puntos de vista (lo político y lo biológico) forman parte de la teoría feminista radical y fueron planteados por Firestone (1976):

\begin{abstract}
Asegurar la eliminación de las clases sexuales requiere que la clase subyugada se alce en revolución y se apodere del control de la reproducción; se restaure a la mujer la propiedad sobre sus propios cuerpos, como también el control femenino de la fertilidad humana [...]. Y así como la meta final de la revolución socialista era no sólo acabar con el privilegio de la clase económica, sino con la distinción misma entre clases económicas, la meta definitiva de la revolución feminista debe ser igualmente no solo acabar con el privilegio masculino, sino con la distinción de sexos misma: las diferencias genitales entre los seres humanos ya no importarían culturalmente. (p. 12)
\end{abstract}

Firestone explica también que la biología es la causante de la opresión de la clase femenina, ya que se encuentra condicionada con la carga de la reproducción. De allí que una característica de este movimiento sea el cuestionamiento de la familia nuclear o biológica. En su opinión, las mujeres solo pueden ser libres aboliendo su función reproductora, implicando así que el ser madre las hace inferiores al hombre (Mujeres Radicales, 2001, p. 34). Por ello, Witting llega a caracterizar «las actividades asociadas a lo femenino, la reproducción, el matrimonio y el cuidado de los hijos, como elementos coercitivos que condicionan socialmente a las mujeres» (Aguilar, 2008, p. 6). Estamos frente a la deconstrucción de lo propiamente femenino.

Por otra parte, se sostiene que la familia y el matrimonio son expresión del capitalismo patriarcal, en el que la mujer no es libre, puesto que «todos son forzados a vivir juntos para que la propiedad pueda ser adquirida e intercambiada y para que la clase dirigente pueda hacer que el trabajo gratuito de las mujeres procree, alimente y cuide a sus actuales y futuros trabajadores» (Mujeres Radicales, 2001, p. 25). Estamos ante la deconstrucción de la familia.

Se rescata como mejor modelo el matriarcado, reconociéndose como «[el] más noble modelo de relaciones humanas igualitarias; las mujeres eran líderes respetadas, los hijos eran criados de manera colectiva e imperaba la libertad sexual, incluyendo la homosexualidad» (Mujeres Radicales, 2001, p. 21). En este sentido, Aguilar (2008) recoge la crítica de Greer respecto de la «mujer eunuco», descrita como un 
«ser producto de la cultura patriarcal, condicionada desde la cuna a la represión de su sexualidad e independencia. Por eso, rechaza el matrimonio y propone la promiscuidad como forma de afianzamiento de la independencia femenina» (p. 6). Estamos ante la deconstrucción del matrimonio.

La deconstrucción de la familia nuclear trae consigo el desconocimiento de la importancia de las relaciones familiares. De hecho, se plantea que a veces los miembros de la familia son obligados a mantener esa estructura patriarcal, siendo los más afectados las mujeres y los niños. Se afirma, entonces, que en la jerarquía de la familia nuclear los hijos se encuentran en la parte inferior, pues no tienen control sobre sus vidas, mentes ni cuerpos, por ello «deben tener la garantía de ser libres de la opresión de las relaciones familiares y los padres deben estar liberados de la responsabilidad única y aislante de criar a sus hijos» (Mujeres Radicales, 2001, p. 48). Posteriormente, este planteamiento tendrá cabida a nivel internacional a partir del desarrollo conceptual del «adultocentrismo» (Unicef, 2013) y las «familias democráticas» (Unicef, 2005).

\section{Neofeminismo y postmodernidad (1980)}

En la década de los 8o, se empieza a cuestionar que el sistema sexo-género deba corresponderse con el sistema naturaleza-cultura, o que género solo tenga que hacer referencia a lo femenino y masculino. La construcción teórica en esta etapa busca romper con el paradigma binario - considerado limitado-y «propone liberar toda manifestación de género que haya sido excluida de la legalidad y reprimida por no participar dentro del binarismo sexual imperante» (Aguilar, 2008, p. 11). En otras palabras, se cuestiona que el sexo -identificado hasta ese momento con lo biológico (permanente y «lo dado») — sea algo natural, y se postula que también se trata de una construcción cultural.

Ya en 1949, Simone de Beauvoir, bajo la pretensión de erradicar la discriminación histórica contra la mujer, había planteado como solución la eliminación de cualquier diferencia biológica entre hombres y mujeres, luego de determinar que las mismas obedecían a patrones enteramente culturales, siendo el dato biológico completamente irrelevante, es decir, no reconoce la existencia de una naturaleza biológica ni psicológica del hombre o la mujer. Así señala:

Ningún destino biológico, psíquico, económico, define la figura que reviste dentro de la sociedad la hembra humana; es el conjunto de la sociedad el que elabora ese producto intermedio entre el macho y el castrado que es calificado como femenino. Solo la mediación del otro puede constituir a un individuo como otro. (citado en Conesa, 2004, p. 46) 
Esto fundamenta una nueva postura del feminismo que pretende alejarse de una concepción que vincule al género (lo cultural y cambiante) con el sexo (lo permanente) por considerarla esencialista. Lo que se propone es que incluso el sexo es culturalmente experimentado. Esto lo explica Saffioti, en cita de Facio y Fries (1999):

\begin{abstract}
Las personas al nacer son transformadas, a través de las relaciones de género en mujeres y hombres, de manera tal que cada una de esas categorías-identidades excluye a la otra. Así, el sexo anatómicamente configurado sugiere, en términos estadísticos, la transformación de ciertos individuos en mujeres y otros en hombre. El convertirse en una y otro es por lo tanto obra de las relaciones de género y no de la biología o la anatomía. (p. 36)
\end{abstract}

Esta nueva teoría de género (llamada también teoría de las relaciones de género) pretende demostrar que la «ideología patriarcal» se esforzó por naturalizar procesos sociales y crear «seres heterosexuales con una identidad de género aprobada por su sociedad, a partir de un proceso social extremadamente complejo y profundamente no natural» (Facio \& Fries, 1999, p. 37). En su lugar, concibe al sexo como una relación vivida y mutable, con una naturaleza relacional, sobre el cual, a su vez, se construye el género (igualmente mutable e histórico). Ya no es solo el patriarcado lo que subyuga a las mujeres (como lo planteaba el feminismo radical), sino que es la «estructura hegemónica del heteropatriarcado» lo que limita y abusa de todas las posibles construcciones de género que puedan existir, no condicionadas al dato biológico. Se empieza - ahora aliado al feminismo- la reivindicación de los derechos LGBTIQ+.

Sobre este tema, Butler (2007) afirmaba que:

El género es una construcción cultural; por consiguiente no es ni resultado causal del sexo ni tan aparentemente fijo como el sexo. Al teorizar que el género es una construcción radicalmente independiente del sexo, el género mismo viene a ser un artificio libre de ataduras; en consecuencia hombre y masculino podrían significar tanto un cuerpo femenino como uno masculino; mujer y femenino, tanto un cuerpo masculino como uno femenino. (p. 6)

De esta manera, si las diferencias son únicamente culturales (dado que, para este planteamiento, lo biológico es irrelevante), la igualdad solo se daría con un profundo cambio cultural de lo que significa «ser mujer». Con ello se presenta «un nuevo modo de concebir la identidad sexual humana, en el que sexo y género llegarán a entenderse como esferas independientes» (Aparisi, 2002, p. 174), pero —este es el cambio- igualmente culturales. 
Así, actualmente pueden verse los efectos de ese modo de pensar cuando se utilizan términos como «sexo asignado al nacer», sexo o género «neutro», o de atribución de derechos en función de «un género asumido», aplicados en la legislación. Tanto el género como el sexo son expresiones culturales que se construyen en relación.

En estos términos, al buscar el reconocimiento de las distintas identidades sexuales (que no se relacionan con lo biológico) y de los derechos que de ellas supuestamente se derivan, se elaboran documentos, como los Principios de Yogyakarta (2007), que - aun siendo un documento no vinculante para los estados- propone la inclusión de términos como orientación sexual, identidad de género, expresión de género, además de sexo, como fundamento para el desarrollo de normas relativas a los derechos humanos.

Esta nueva concepción del término género plantea una construcción de la identidad sexual y de género tal que lleva a afirmar que «los géneros masculino y femenino, serían una construcción de la realidad social que deberían ser abolidos. [...] [y] que los sexos ya no son dos, sino cinco» (Fernández, 2008, p. 11), o más, de acuerdo con la construcción cultural.

\section{Contenido del enfoque de género}

Lo dicho hasta aquí nos puede dar una idea del contenido del término género: una construcción cultural disociada del sexo biológico (que también es una vivencia construida) y que depende enteramente de la subjetividad del individuo; circunscrito en una idea fuertemente arraigada de que lo más importante es la plenitud del aspecto sexual (en el sentido de placer) y que, en el ámbito de la búsqueda de la igualdad, se impedirá cualquier intento de distinción entre hombres y mujeres, entre lo masculino y lo femenino.

Este contenido (amplio y confuso) se ha visto reflejado en distintos documentos nacionales e internacionales bajo el llamado «Enfoque de género», enarbolado como la búsqueda de igualdad entre hombres y mujeres, pero que finalmente es la teoría que sustenta que la identidad, la sexualidad, la reproducción y el poder no están determinados ni por la biología ni la naturaleza. Todo es cultura y «libertad». A ese respecto, se ha pronunciado el Tribunal Constitucional peruano (2015) en su sentencia que autoriza el cambio de sexo en el documento de identidad:

Así las cosas, la realidad biológica, a tenor de lo expuesto, no debe ser el único elemento determinante para la asignación del sexo, pues este, al ser también una construcción, debe comprenderse dentro de las realidades sociales, culturales e interpersonales que 
la propia persona experimenta durante su existencia. Por ende, el sexo no debe siempre ser determinado en función de la genitalidad, pues se estaría cayendo así en un determinismo biológico, que reduciría la naturaleza humana a una mera existencia física, y ello obviaría que el humano es un ser también psíquico y social. (p. 7) [el subrayado es nuestro]

Cabe señalar que, a nivel internacional, desde siempre se trabajó en términos de buscar la igualdad entre mujeres y hombres, y así se expresa en la Convención de 1979 sobre la eliminación de todas las formas de discriminación contra la mujer y en las siguientes. Es recién en la IV Conferencia Mundial sobre la Mujer (Beijing 1995), donde se menciona por primera vez el término «perspectiva de género», considerando que:

El género se refiere a los roles y responsabilidades de la Mujer y el Hombre que son determinados socialmente. El Género se relaciona con la forma en que se nos percibe y espera que actuemos como mujeres y hombres, por la forma en que la sociedad está organizada, no por nuestras diferencias biológicas. (Consejo Pontificio para la Familia, 2004, p. 581)

Hasta aquí, si bien se considera el género como dependiente de un constructo social, no se desechan las diferencias biológicas y se mantiene en el plano de la búsqueda de la equidad entre hombres y mujeres. Sin embargo, no debemos olvidar que en la base del feminismo está considerar que estos «roles» son en sí mismos injustos en la medida en que fueron diseñados por los hombres desde su posición dominante:

Como los hombres han tenido el poder de definir las cosas y los valores, caso sólo las cosas y los valores que ellos han definido están aceptadas como válidas en nuestra cultura y por ende, esta cultura es masculina o al menos, predominantemente masculina. (Facio \& Fries, 1999, p. 50)

Por lo señalado, para delimitar el contenido del enfoque de género, debemos tener en cuenta lo siguiente:

- Parte de la idea de que existe una histórica desigualdad entre hombres y mujeres, producto del poder sexual y económico ejercido por los primeros en contra de las segundas. Esta desigualdad da lugar a la asignación de unos roles que, al estar relacionados con los rasgos biológicos, suponen una desventaja para la mujer, que también se manifiesta en situaciones de violencia.

Kimmel, en cita de Vivanco (2006), señala que: 
Esta es la definición de lo que llamaremos masculinidad hegemónica, la imagen de masculinidad de aquellos hombres que controlan el poder, que han llegado a ser la norma de las evaluaciones psicológicas, en la investigación sociológica y en la literatura de autoayuda [...]. Igualamos la masculinidad con ser fuerte, exitoso, capaz, confiable y ostentando control. Las propias definiciones de virilidad que hemos desarrollado en nuestra cultura perpetúan el poder que unos hombres tienen sobre otros, y que los hombres tienen sobre las mujeres. (p. 153)

- Los femenino y masculino son producto de una construcción cultural que no tiene que ver con el sexo biológico, antes bien, es la causa de desigualdad y trato discriminatorio contra la mujer:

[...] la discriminación estructural contra las mujeres tiene en su base un conjunto de patrones socioculturales discriminatorios - que privilegian lo masculino sobre lo femenino- reforzando la asignación desigual de roles [...] que infravalora lo femenino en comparación con lo masculino. [...] dichos patrones se reproducen y perpetúan por la acción de las normas y cultura institucional que refuerzan la situación de inferioridad o subordinación de las mujeres en la sociedad. (Ministerio de la Mujer y Poblaciones Vulnerables [MIMP], 2019, p. 12)

- La identidad sexual del sujeto es algo libremente construido en base a lo que él considera útil y bueno para sí mismo, en ejercicio de su libertad sexual. Por tanto, el dato biológico no resulta relevante para la formación de dicha identidad. Esto supone la exaltación de la sexualidad vinculada únicamente a la obtención de placer.

- Los rasgos biológicos y sexuales - como la maternidad- son la principal causa de exclusión de la mujer, por lo que la «liberación» (que genera igualdad) solo se logrará cuando ella tenga el absoluto control de su cuerpo en materia reproductiva. Por ello, se plantea el aborto como derecho y la «libertad sexual» $\sin$ límites y sin consecuencias:

Los derechos reproductivos no se agotan en la capacidad de la mujer para elegir cuándo quiere tener relaciones sexuales con un hombre y cuándo quiere que, producto de ellas, nazca descendencia, sino que van mucho más allá: es también fundamental que, tanto el hombre como la mujer, se liberen de la necesidad de la previa relación sexual para poder procrear. (Vivanco, 2006, p. 161)

- El matrimonio y la familia son construcciones sociales que abonan la opresión de la mujer, porque le imponen un rol social posiblemente no querido y son el principal obstáculo para la realización personal de la mujer. 
Así lo explica Riddiough (en cita de Fernández, 2008):

La familia nos da las primeras lecciones de ideología de clase dominante y también le imparte legitimidad a otras instituciones de la sociedad civil. Nuestras familias son las que nos enseñan primero la religión, a ser buenos ciudadanos, tan completa es la hegemonía de la clase dominante en la familia, que se nos enseña que ésta encarna el orden natural de las cosas. Se basa en particular en una relación entre el hombre y la mujer que reprime la sexualidad, especialmente la sexualidad de la mujer. (p. 14)

- Si se admite la existencia de la familia como una institución humana más de convivencia, será siempre y cuando las relaciones dentro de ella se encuentren libres de estructuras o jerarquías (que se entendía generalmente como el reconocimiento de la autoridad de los padres), para ser más bien estructuras democráticas, en las que cada individuo pueda alcanzar su pleno desarrollo individual en condiciones igualitarias:

Se busca el establecimiento de relaciones libres e igualitarias entre los individuos y de sistemas de autoridad que no estén fijados mediante contratos rígidos basados en la complementariedad de roles, sino en la especialización de cada uno, de acuerdo con las capacidades de cada persona y teniendo en cuenta las posibilidades que cada persona tiene para desarrollarse, más allá de ser hombre o mujer. (Unicef, 2005, p. 8)

- Si bien el rol de la mujer en la sociedad debe ser deconstruido, también debe suceder lo mismo con el varón. Así, en la relación varón-mujer, se plantea en términos de «nuevas masculinidades», en las que deben ser abandonadas las características propias del «ser masculino». Esto justifica, por otro lado, que el papel del padre o de la madre sean absolutamente prescindibles, dando lugar a que cualquier tipo de relación permita la formación de una familia o se convierta en un espacio adecuado para la crianza de los niños.

Es ilustrativo al respecto lo que señal Jagger (citado en Conesa, 2004):

El final de la familia biológica eliminará también la necesidad de la represión sexual. La homosexualidad masculina, el lesbianismo y las relaciones sexuales extramaritales ya no se verán en la forma liberal como opciones alternas, fuera del alcance de la regulación estatal [...] en vez de esto, hasta las categorías de homosexualidad y heterosexualidad serán abandonadas: la misma «institución de las relaciones sexuales» en que hombre y mujer desempeñan un rol bien definido, desaparecerá. La humanidad podría revertir finalmente a su sexualidad polimorfamente perversa natural. (p. 46) 
Siguiendo a Aguilar (2008), cabe preguntarnos si las diferencias de género sostenidas por el feminismo a lo largo de su historia sirven para el proyecto de lograr la igualdad efectiva entre hombres y mujeres o, por el contrario, la teorización de la construcción social de la identidad sexual ha supuesto una deformación en los principios que ahora mismo lo sumen en un callejón sin salida que no ofrece soluciones en favor de la igualdad, ni para la mujer ni para la familia.

\section{Influencia del enfoque de género en las políticas públicas familiares. El caso peruano}

El Estado peruano ha definido el Enfoque de género en diversas normas legales, planes y guías de políticas públicas. Sin pretender citar a todos, haremos referencia a los documentos más representativos.

El Ministerio de la Mujer y Poblaciones Vulnerables (2014) lo define como:

[...] la forma de mirar la realidad identificando los roles y las tareas que realizan las mujeres y los hombres en una sociedad, así como las asimetrías y relaciones de poder e inequidades que se producen entre ellas y ellos, permitiendo conocer y explicar las causas que las producen para formular medidas (políticas, mecanismos, acciones afirmativas, normas, etc.) que contribuyan a superar las brechas sociales producidas por la desigualdad de género. (p. 6)

La Política Nacional de Igualdad de Género (MIMP, 2019, s. p.) conceptualiza el término género:

[...] como las identidades, las funciones y los atributos construidos socialmente de la mujer y el hombre, así como al significado social y cultural que la sociedad atribuye a esas diferencias biológicas, situación que da lugar a relaciones jerárquicas entre hombres y mujeres en las que se distribuyen facultades y derechos en favor del hombre y en menoscabo de la mujer.

Finalmente, el Plan Nacional Contra la Violencia de Género 2016-2021 (MIMP, 2016, s. p.) sostiene que dicho enfoque:

Pone en evidencia desigualdades sociales y relaciones asimétricas de poder de varones y mujeres, las cuales han determinado históricamente la subordinación de las mujeres, la violencia contra ellas y limitado sus posibilidades de realización y autonomía. Señala que roles, atributos, comportamientos, posiciones jerárquicas, asumidos de manera distinta y excluyente por hombres y mujeres no son naturales, sino construidos social y culturalmente. Recusa la división sexual del trabajo, entre el ámbito productivo, asignado a los 
hombres, y reproductivo, a las mujeres, que crea desventajas para ellas e impiden su plena realización y participación en el bienestar, y propugna las responsabilidades compartidas de hombres y mujeres en los espacios públicos y domésticos [...].

Puede notarse que en estas definiciones se entiende que los roles asumidos por varones y mujeres son estrictamente culturales y desconocen cualquier tipo de asociación con el aspecto biológico. Vincula el aspecto reproductivo de las mujeres con una posición de desventaja objetiva frente al varón, que impide su plena realización y bienestar. Esto implica un serio cuestionamiento a la maternidad o, cuando menos, una connotación negativa asumida desde el ámbito del propio Estado. Hace hincapié en que las desigualdades se presentan únicamente por los roles asignados por la sociedad y reconoce a la mujer como sujeto aislado (y desvinculado de la familia o de la pareja).

Resultaría altamente contradictorio que un planteamiento teórico que, de forma abierta, se opone a la familia pudiera contribuir con su fortalecimiento o protección. Prueba de ello es que, pese a los años que lleva implementándose el enfoque de género en las políticas públicas en el Perú, se observa que el ingreso promedio de las mujeres representa el $68 \%$ del de los hombres (y se ha mantenido constante en los últimos años); que la fecundidad en el país ha disminuido en 3.8\% (las mujeres optan por tener menos hijos); que la media de edad de la maternidad en las mujeres ha aumentado; que el número de divorcios se ha incrementado en $11,2 \%$; que hay más mujeres unidas en convivencia (36.1\%) que casadas (21.4\%); que los hogares monoparentales van en aumento; que cada día al menos cuatro adolescentes menores de 15 años queda embarazada, y que la violencia contra la mujer no ha disminuido (68.2\%) (Instituto Nacional de Estadística e Informática [INEI], 2016). Es decir, respecto a las situaciones que involucran a la familia, las condiciones se han mantenido igual. En todos los casos, no existe mejora.

Sin embargo, y para profundizar en esta premisa, realizaremos un breve análisis de las normas relacionadas con la familia - en las que se incluye el enfoque de género- que se han aprobado en los últimos años. Para ello, hemos identificado algunos aspectos a considerar: cambios en el lenguaje, desigualdad y no discriminación, violencia familiar, salud sexual y reproductiva, fortalecimiento del matrimonio y la familia y educación.

\section{Cambios en el lenguaje}

Una de las acciones ineludibles para lograr la igualdad efectiva y la visibilización de la mujer - en términos del enfoque de género- es el cambio de los parámetros usuales del lenguaje. Así, a la deconstrucción de la identidad se suma la decons- 
trucción del lenguaje. Esto, básicamente, porque plantean que el lenguaje (de la misma forma que el Derecho) se ha desarrollado a partir de la hegemonía masculina, de tal manera que refleja la dominación del hombre sobre la mujer.

Esto se evidencia en la Guía para el uso inclusivo del lenguaje en las entidades públicas, documento elaborado por MIMP (2014):

El lenguaje desempeña un rol determinante para la socialización y transmisión de la cultura, creando realidades y formando identidades. Su uso adecuado, a través de un lenguaje inclusivo, puede promover cambios positivos entre las personas logrando que actitudes sexistas y discriminatorias se conviertan en prácticas equitativas, respetuosas e inclusivas que visibilicen la presencia de la mujer. (p. 5)

En dicha guía se propone, además del abandono del uso del masculino gramatical como género no marcado, dejar de utilizar términos que promueven estereotipos —como «señorita», «y esposa» (para el caso de invitaciones)—, abandonar el sexismo en la redacción (no asimilar el término «mujer» con el término «esposa») y eliminar del uso corriente del lenguaje palabras discriminatorias, como «caballerosidad», «hombría», «instinto maternal» o «esencia femenina». Eliminar el uso del término hombre (para referirse a ambos sexos) y expresiones androcéntricas, entre otros.

La pretensión de eliminar expresiones como «instinto maternal» 0 «esencia femenina» nos recuerdan la especial relación que tienen la teoría de género y la familia. Así, uno de los objetivos de dicha teoría, como se ha evidenciado, es la deconstrucción de cualquier estructura que implique relaciones duraderas y estables, en las que se distingan claramente los roles femeninos y masculinos, es decir, madre y padre, y en la que los hijos se encuentren protegidos bajo la patria potestad (y autoridad) de sus padres. Por ello, consideramos altamente probable que cualquier política pública dirigida a la familia con contenido de enfoque de género resulte perjudicial o, cuando menos, inútil.

\section{Desigualdad y no discriminación}

Cuando a nivel de políticas públicas se habla de igualdad, se debe pensar que lo que se busca es una igualdad plena y real de oportunidades entre todos los individuos. En este caso, una igualdad efectiva entre hombres y mujeres (que, por otro lado, ya se encuentra garantizado por las normas jurídicas). Desde otra perspectiva, esto no nos debe llevar a desconocer que existan diferencias biológicas, naturales o sexuales que, a nivel jurídico, en nada tendrían que afectar la convivencia de ambos sexos. Entre las normas con enfoque de género emitidas en este rubro tenemos: 


\section{La Política Nacional de Igualdad de Género. Decreto Supremo № 008 -2019- MIMP (2019)}

Esta norma desarrolla conceptos como discriminación estructural, que se refiere al conjunto de dinámicas reproducidas por patrones socioculturales y se expresa en prácticas y discursos excluyentes y violentos avalados por el orden social; distribución desigual del poder, como causa de patrones socioculturales discriminatorios tolerados socialmente, compuesta por costumbres, creencias, prejuicios y dogmas religiosos en relación a lo que una mujer y un hombre deben ser y hacer, y que explican cómo, históricamente, la organización patriarcal determinó una cultura del privilegio de lo masculino sobre lo femenino. Considera discriminatorio la referencia a patrones de feminidad o masculinidad, señalando que la familia es la que los reproduce.

Creemos que, por el punto de partida adoptado, difícilmente se puede llegar a promover una situación de convivencia en igualdad (sobre todo si se considera la influencia negativa de la familia) donde hay un culpable y una víctima, al parecer, irreconciliables.

Un tema importante es que el índice de Igualdad de Género para el 2016 fue de 0.391, siendo lo óptimo (la igualdad perfecta) el rango o (MIMP, 2017, p. 19). En este sentido, cabe preguntarse si plantear la situación actual en términos de desigualdad y discriminación estructural e histórica no es más bien una distorsión de la realidad.

\section{Violencia contra la mujer y familia}

La violencia familiar se configura como un grave problema en el Perú. Entre enero y junio de 2018, la Policía Nacional da cuenta de 106421 denuncias por violencia familiar; más de la mitad (51.5\%) fueron por agresión física; $42.9 \%$, por violencia psicológica, y 5,6\%, violencia sexual (INEI, 2018, p. 10). Sin embargo, desde el año 2009 (76.95\%) al 2017 (65.4\%), los casos de violencia contra la mujer solo han disminuido en un $11.5 \%$, pese a la gran cantidad de normas y acciones políticas con enfoque de género que se han implementado, lo que podría llevarnos a afirmar que las políticas públicas relacionadas con este aspecto no están funcionando.

Una de las razones podría ser que el Plan y las políticas de género se centran únicamente en identificar las causas de la violencia con los patrones o roles culturales aprendidos y que se desarrollan primero en la familia (MIMP, 2016), lo cual supone un prejuicio propio de la teoría de género. De esta forma, no se considera la posibilidad de que la familia también pueda ser parte de la solución en la erradicación de 
la violencia. No existe ninguna referencia en las normas al trabajo estructurado que se podría hacer desde las familias para disminuir los casos de agresión.

\section{Ley $N$ ․o 30364. Ley para prevenir, sancionar y erradicar la violencia contra las mujeres y los integrantes del grupo familiar (2015)}

La norma distingue las situaciones de violencia contra la mujer (art. 5.ํ) de aquellas contra los integrantes del grupo familiar, como si se tratara de situaciones distintas o, en todo caso, privilegiando injustamente la protección a la mujer, estableciendo categorías innecesarias para combatir la violencia sin considerar que dentro de la familia incluso podemos encontrar personas con mayor vulnerabilidad (niños, adultos mayores y personas con discapacidad).

Ante la complejidad del problema, no puede perderse de vista que (I) la mayoría de casos de violencia contra la mujer se verifican en un entorno familiar; (II) no se puede descuidar los otros casos de violencia familiar por priorizar la atención a la violencia contra la mujer, y (III) la rehabilitación de las víctimas requiere, necesariamente, del entorno familiar para que sea efectivo.

Mención aparte merece el concepto de «violencia económica», que se introduce en la norma, pero que desconoce la dinámica y realidad de cada familia, propiciando que este tipo de violencia pueda ser entendida de manera subjetiva por cualquier miembro de la familia e incluso calificar como situación de violencia aquella que es, en realidad, una situación propia de la convivencia en familia. Posiblemente, el hecho de que deba ser un juez quien determine esta situación cauce mayor perjuicio que el que se busca solucionar con la norma en cuestión. Sin negar que este tipo de violencia se presente en algunos entornos familiares (principalmente en aquellos donde la mujer no tiene un trabajo fuera del hogar), consideramos que es importante establecer criterios claros para su calificación.

\section{Decreto legislativo que fortalece la lucha contra el feminicidio, la violencia familiar y la violencia de género. Decreto Legislativo 1323 (2017)}

La norma modifica diversos artículos del Código Penal, entre ellos el art. 46. ${ }^{\circ}$, para considerar como circunstancia de agravación de la pena: «d) Ejecutar el delito bajo móviles de intolerancia o discriminación, tales como el origen, raza, religión, sexo, orientación sexual, identidad de género [...]». Con ello, no solo introduce como categorías de discriminación la orientación sexual y la identidad de género que, hasta la fecha, no tenían reconocimiento explícito en las normas peruanas, sino que - al encontrarse en la parte general de la norma - la causal agravante se aplica para cualquier delito. Así, como señala Balmaceda (2017), «estafar, matar, injuriar, 
realizar trata de personas, etc., tendrán penas mucho más graves si el sujeto pasivo es miembro del colectivo LGTBIQ+. Y esto queda fuera de toda proporción» (p. 45). Por otro lado, modifica el delito de discriminación contenido en el art. 323. ${ }^{\circ}$ del Código Penal, y sanciona los:

Actos de distinción, exclusión, restricción o preferencia que anulen o menoscaben el reconocimiento, goce o ejercicio de cualquier derecho de una persona o grupo de personas reconocido en la ley [...] basados en motivos raciales, religiosos, nacionalidad, edad, sexo, orientación sexual, identidad de género [...]. [La cursiva es nuestra]

La crítica a esta modificación radica en que en la descripción extensiva de los «actos de discriminación», donde se incluye hasta la acción de distinguir, se anula el primer principio del Derecho, la justicia, que implica darle a cada uno lo que le corresponde, lo que al mismo tiempo conlleva una comprensión del trato justo como aquel que distingue entre las diferencias de las personas (Balmaceda, 2017, p. 43). En otras palabras, el «trato igualitario» que promueve el enfoque de género, en la práctica, debería entenderse como un trato equitativo, es decir, buscando la igualdad con justicia, lo que supone también, en algunos casos, tratar de manera distinta (piénsese en las licencias por maternidad, por ejemplo). No obstante, con una norma que considera delito el trato distinto, es probable que se presenten serias cuestiones de interpretación.

\section{Salud sexual y reproductiva}

Uno de los tópicos del enfoque de género es la promoción de los derechos sexuales y reproductivos, caracterizados por el libre acceso a medios de anticoncepción (incluido el aborto) a cualquier edad y la vivencia placentera de la sexualidad, sin relacionarla con vínculos o afectos.

Entre los objetivos de las políticas de salud reproductiva está la reducción de los embarazos de adolescentes. Pero, como suele suceder con otros aspectos, los datos estadísticos demuestran que los mismos no han disminuido y se mantienen a una tasa constante del 13\% (adolescentes entre 15 y 19 años de edad).

Las causas de este tema son sumamente complejas y multisectoriales (Mendoza \& Subiría, 2013), por lo que mal haría en reducirse solo a patrones y roles culturales, y mucho menos se soluciona únicamente garantizando el acceso a métodos anticonceptivos y a educación sexual.

Se requieren medidas más complejas, como promover la postergación del inicio de la actividad sexual en la adolescencia, el involucramiento de los padres en la forma- 
ción de sus hijos, asegurar espacios de sana recreación para los adolescentes y, por supuesto, el acceso a una educación en la sexualidad relacionada con el compromiso, entre otros. Los resultados seguirán siendo desfavorables en la medida en que se reduzcan a promocionar la vivencia libre y placentera de la sexualidad o en cómo hacer para limitar o eliminar los riesgos de la actividad sexual a edad temprana (uso de métodos anticonceptivos e incluso aborto).

Norma Técnica de Salud de Planificación Familiar N.o 124-2016-MINSA/V.o1, aprobada por Resolución Ministerial № 652-2016/MINSA, modificada por Resolución Ministerial N.o 536-2017/MINSA (2017)

La norma promueve el «acceso de la población a los diferentes métodos anticonceptivos [...] para lograr el bienestar y la autonomía de las mujeres» (p. 11). Busca brindar «atención integral con énfasis en salud sexual y reproductiva en servicios diferenciados para los adolescentes» (p. 14), a quienes «se les brindará todos los servicios».

No hace referencia a que, por la edad, los servicios dirigidos a adolescentes deberían - cuando menos - tener la autorización de sus padres. Señala que «la edad no es razón suficiente para descartar ningún método anticonceptivo, pero los aspectos socioconductuales de los adolescentes deben ser considerados, siendo muy importante valorar su aceptabilidad y motivación» (p. 93). No menciona la necesidad de que los padres tengan conocimiento de la atención de sus menores hijos a través del programa.

La norma incluye como método anticonceptivo el uso de Levonogestrel (AOE), que puede ser entregado tanto a adultos como a adolescentes (de cualquier edad y sin conocimiento de sus padres) y no se señala especial atención a la comunicación de los efectos por su uso reiterado.

En el marco del enfoque de género, esta norma se justifica debido a que se tiene que garantizar el acceso al ejercicio de la sexualidad sin tener que asumir las consecuencias naturales de ello (embarazo). Cabe señalar que de los tres efectos que tiene el AOE, uno de ellos es claramente abortivo, ya que impide la implantación del embrión en el útero. Sin embargo, por cuestiones estrictamente de interpretación normativa, la Corte Interamericana de Derechos Humanos (Corte IDH, 2012) consideró que la concepción debía entenderse como el momento de la implantación del embrión en el útero. Así, antes de la implantación, el embrión no se encuentra en los términos de protección (del derecho a la vida) que otorga la Convención. Esto justifica que el Estado peruano continúe con la distribución del AOE y, por tanto, con la aplicación de sus políticas. El uso del AOE se contradice con el hecho que la propia norma establece que «el aborto no es considerado un método de planificación familiar» (p. 30). 
Cabe señalar que los indicadores de la norma se encuentran orientados a medir la reducción de la tasa de fecundidad.

Guía Técnica Nacional para la Estandarización del Procedimiento de la Atención Integral de la Gestante en la Interrupción Voluntaria por Indicación Terapéutica del Embarazo menor de 22 semanas con consentimiento informado en el marco de lo dispuesto en el artículo 119. del Código Penal. RESOLUCIÓN N.o 486-2014/MINSA (2014)

En el marco de la regulación de los derechos sexuales y reproductivos — con enfoque de género- se aprobó la guía que «regula la procedencia de un aborto cuando se encuentra en grave peligro la vida y la salud de la gestante» (p. 8).

Una de las cuestiones señaladas en esta guía es que el aborto se realiza «ante la petición de la gestante» (art. 6, inc. 2.2), la misma que será revisada por una Junta Médica (art. 6, inc. 2.4) que podrá aprobar o denegar el procedimiento abortivo. Si la Junta lo deniega, la gestante puede insistir en su petición a fin de que la negativa sea revisada por una nueva Junta Médica (art. 6, inc. 3.4). Llama la atención esta suerte de «apelación» de la decisión médica, como si el fin de la solicitud fuese el aborto mismo y no la salud o vida de la gestante. Por otro lado, la guía denomina «contenido uterino» al embrión o feto antes de las 22 semanas de gestación (art. 6.7).

Una de las cuestiones que más polémica ha generado esta guía es que el numeral 11 señala como causa justificante «cualquier otra patología materna que ponga en riesgo la vida de la gestante o genere en su salud un mal grave y permanente, debidamente fundamentada por la junta médica» (art. 6). Esto, a decir de Pacora-Portella (2014), «es una puerta de entrada para introducir legalmente el aborto eugenésico y el aborto del embarazo no planeado o deseado, debido a que la madre está perturbada psicológicamente o ha sido víctima de abuso sexual» (p. 238). Tal es el caso de una mujer que, al ser informada por su médico que su hija tenía el Síndrome de Turner, solicitó que se le realizara un aborto terapéutico. El médico se negó a realizar el procedimiento «en la medida que la patología existente no afectaba la vida de la gestante» (Instituto Nacional de Defensa de la Competencia y la Propiedad Intelectual [Indecopi], 2017, p. 3). Pese a ello, la mujer consultó a su psiquiatra, quien le diagnosticó una depresión recurrente con ideas suicidas, aparentemente desencadenadas por la gestación, recomendando la interrupción del embarazo. Con este nuevo diagnóstico, acudió a su clínica para solicitar el aborto terapéutico, petición que le fue denegada. Por este hecho, la clínica fue sancionada con una multa por falta de idoneidad en el servicio.

Como este, no dejarán de presentarse casos en los que, en lugar de establecer como principio el cuidado de la vida y la salud de la madre y el niño, se busque la forma de 
realizar un aborto a pedido de la gestante, sobre todo porque la experiencia médica devela que «la interrupción médica del embarazo solo se justifica en casos poco frecuentes» (Pacora-Portella, 2014, p. 239), menos que aquellos que menciona la guía técnica.

\section{Política Nacional de Igualdad de Género. Decreto Supremo N.o oo8-2019- MIMP (2019)}

Sostiene que «en el Perú persisten normas, políticas y prácticas discriminatorias sobre las esferas de la sexualidad y la reproducción que afectan de manera decisiva la vida de las mujeres, exponiéndolas a graves riesgos, daños y desventajas» (p. 10). $\mathrm{Al}$ realizar el diagnóstico sobre temas de salud reproductiva afirma que «las limitaciones en el acceso de las/os adolescentes a información y métodos anticonceptivos al iniciar una vida sexual activa incrementan la probabilidad de un embarazo no deseado en la adolescencia» (p. 17). Señala como objetivos específicos:

(i) [R]educir la violencia hacia las mujeres; (ii) garantizar el ejercicio de los derechos a la salud sexual y reproductiva de las mujeres; (iii) garantizar el acceso y participación de las mujeres en espacios de toma de decisiones; (iv) garantizar el ejercicio de los derechos económicos y sociales de las mujeres; (v) reducir las barreras institucionales que obstaculizan la igualdad en los ámbitos público y privado entre hombres y mujeres, y (vi) reducir la incidencia de los patrones socioculturales discriminatorios en la población. (pp. 31-34)

Como se puede apreciar, aun cuando se podría esperar una norma dirigida a hombres y mujeres, esta se centra exclusivamente en la mujer, de manera individual y sin colocarla en ningún contexto específico (familia, relación de pareja, trabajo, etc.). Se busca la igualdad por la igualdad, la libertad por la libertad, a partir de la identificación de un responsable primario de las desventajas que las mujeres sufren.

\section{Promoción del matrimonio y la familia}

Este aspecto es quizás el más afectado por las políticas con enfoque de género, pues si se considera a la familia como el origen de las desigualdades, poco o nada se hará por mantener y proteger sus estructuras, lo que incluye claramente la promoción del matrimonio como medio primario de convivencia con el fin de dar origen a la familia. Al contrario, se promueven diversas formas de convivencia, diversas estructuras a las que también se llaman «familiares», desconociendo que la familia (la estructura que vincula a las personas no solo a través de los lazos afectivos, sino también de consanguinidad e intergeneracionalidad) no puede tener un tratamiento similar a otras formas de convivencia. Esto es así porque una de sus particu- 
laridades es que conlleva la visión de «don», que se deriva del hecho de que en ella no puede haber nunca un sujeto aislado, sino definido en relaciones. En la familia se es por los otros. Sostiene Melina (2015) que el padre siempre es «padre de»; él no solo «tiene» un hijo, sino que «es» padre. Por ello, en la familia, el bien del otro no se concibe en competencia con su propio bien, como si se tratara de un conflicto de intereses; en ella no existen «relaciones negociadas», «bien individual» o «igualitarismo», dado que antes de perseguir el bien individual se promueve la búsqueda del bien del otro.

\section{Decreto Legislativo para el fortalecimiento y la prevención de la violencia en las familias. DL 1408 (2018)}

La publicación del Decreto Legislativo N. 1408 tiene como finalidad «contribuir al fortalecimiento de familias democráticas, de manera que se consideren los intereses y necesidades de todas/os sus integrantes en la toma de decisiones, y propiciando relaciones igualitarias, inclusivas, respetuosas y libres de violencia [...]» (s. p.). Incluso cuando no existía motivo, también supuso la derogatoria de la Ley N. 28542 (Ley de fortalecimiento familiar, 2005), pese a que después de la protesta ciudadana, vía modificación, se incluyeron varios de sus artículos en la nueva norma (menos aquellos referidos al fortalecimiento del matrimonio y la inclusión de la perspectiva de familia en las políticas públicas).

La norma enumera los deberes del Estado para el fortalecimiento de la familia en el marco de la lucha contra la violencia familiar. Sin embargo, de su lectura se puede apreciar que promueve el individualismo (al ponderar el interés individual de los miembros de la familia), se desconoce la diferencia de los roles que se asumen con la maternidad y la paternidad. Al equipararlos, los vacía de contenido y los hace prescindibles. Un ejemplo de esto es que en toda la norma no se utilizan las palabras padre, madre o hijos; en su lugar se usa «miembros», «integrantes»y «progenitores».

La norma presupone que las relaciones familiares se basan en la «negociación», en lugar del amor o la búsqueda del bien común. Se evidencia que busca el «empoderamiento» de la mujer en detrimento de los demás miembros. Parte de la premisa errónea de que todas las relaciones en la familia, por ser asimétricas, son negativas, con lo cual se desconoce la autoridad de los padres, distorsionando el concepto de familia democrática, considerada, hasta este momento, como estilo educativo (Torío, Peña \& Rodríguez, 2008). 


\section{Plan Nacional de Fortalecimiento a las Familias 2016-2021 (MIMP 2016a)}

Incorpora el enfoque de género para:

[...] analizar desde una mirada integral los efectos de las desigualdades sociales y relaciones asimétricas de poder, en la calidad de vía de hombres y mujeres y cómo se perpetúa desde las familias, como en el desigual ejercicio de las responsabilidades familiares y que repercuten en la conciliación de la vida familiar y el trabajo. (p. 24)

Los objetivos del plan son: (I) garantizar la promoción, protección y fortalecimiento de las familias; (II) promover el derecho a formar y vivir en familia de toda persona contribuyendo a su desarrollo y bienestar integral; (III) fomentar las responsabilidades familiares compartidas y de la conciliación entre la vida familiar y el trabajo; (IV) fomentar las relaciones familiares democráticas y asertivas como mecanismo para la prevención de la violencia familiar, y (V) generar las condiciones para la satisfacción de las necesidades básicas en las familias.

Aun cuando podemos coincidir con los objetivos del plan, los indicadores refieren que el mismo no ha sido plenamente ejecutado y no se han cumplido con los indicadores señalados. Probablemente se deba también a las mismas razones por las que el plan anterior (2011-2014) no se ejecutó plenamente: al parecer no hay disposición de autoridades para involucrarse con la familia. Esto puede verse en los informes de resultados que emite el ente rector (MIMP) sobre la ejecución de este plan: los temas referidos a fortalecimiento familiar, infraestructura, promoción del empleo y corresponsabilidad no tuvieron los resultados esperados. Así, «de los 34 indicadores priorizados en el año 2016, 12 acumulan menos del $100 \%$ de la meta programada 2017 y 14 indicadores tienen o \% de cumplimiento de la meta programada 2017» (MIMP, 2017, p. 77). Por otro lado, el Plan Nacional de Fortalecimiento a las Familias 2016-2021 ha sido calificado como un instrumento con «débiles condiciones técnicas en el planteamiento de sus indicadores» (MIMP, 2019, p. 1). Cabe resaltar que el documento señala que, de los 81 indicadores con los que cuenta el Plan, solo se evaluarán 8; por lo que no se contará con información concreta del avance de su ejecución.

\section{Ley 292227. Ley que regula el Procedimiento No Contencioso de la Separación Convencional y Divorcio Ulterior en las Municipalidades y Notarías (2008)}

Esta norma, lejos de promover el matrimonio (el art. $4 .^{\circ}$ de la Constitución Política del Perú [CPP] establece la promoción del matrimonio como un deber del Estado), regula el llamado «Divorcio Rápido», mediante el cual una pareja puede disolver su 
vínculo matrimonial en un plazo de 2 a 3 meses. La norma únicamente responde al alto número de divorcios en la sociedad y, por supuesto, no pretende ser un elemento disuasivo del mismo.

\section{Plan Nacional de Acción por la Infancia y la Adolescencia 2012-2021. PNAIA 2021. Decreto Supremo N.o oo1-2012-MIMP (2012)}

El plan acoge el enfoque de género señalado que:

[...] permite poner en evidencia desigualdades sociales y relaciones asimétricas de poder de varones en detrimento de las mujeres de cualquier edad, construidas sobre las bases de diferencias biológicas en el plano de lo sexual. Plantea desterrar cualquier relación jerárquica basada en estas diferencias. (p. 17)

Hace referencia al ámbito privado de la familia y señala que por dicho ambiente «un alto número de niñas y niños ve afectada su integridad física o sexual y se vulneran sus derechos fundamentales». Luego, en un contexto negativo, se refiere a la familia como el lugar «donde también niñas y niños naturalizan e interiorizan valores autoritarios, discriminadores por diferencias de género, de etnia, de raza, de edad, de estrato social y de orientación sexual».

No obstante, la norma también reconoce que «el ámbito familiar [es] el contexto más propicio para implementar políticas que generen vínculos afectivos significativos, que favorezcan una socialización que promueva valores democráticos y el respeto a los derechos y estimulen el desarrollo humano» (p. 15). Pero, a su vez, promueve el concepto de «familia democrática», determinando que «la familia no debe ser una estructura jerárquica y autoritaria sino una institución democrática en donde prevalezca el respeto mutuo, libre de todo tipo de violencia y donde se apoye el desarrollo de cada uno de sus integrantes» (p. 16). Se tergiversa con ello la noción de autoridad que - guiados por el amor- deben tener los padres, y se utiliza el término en un sentido negativo.

Se ocupa, además, de los derechos sexuales y reproductivos, por lo que uno de sus indicadores es:

Propiciar, desde el Ministerio de Salud, el acceso a información y métodos anticonceptivos oportunamente, así como la capacitación de las y los adolescentes para que puedan ejercer una actividad sexual responsable, prioricen su educación y posterguen la maternidad/paternidad hasta la edad adulta. (p. 75) [el subrayado es nuestro] 
Cuando la familia tiene una connotación tan negativa en una política, difícilmente se podrá considerar como actora del cambio social, y su papel será relegado a mera espectadora de los cambios que se presentan en la sociedad, aunque le afecten.

\section{Educación}

La influencia del enfoque de género en la educación es evidente. En este caso, la norma que se analiza es el Currículo Nacional.

\section{Currículo Nacional de la Educación Básica. Resolución Ministerial № 281-2016-MINEDU (2016)}

Contiene el enfoque de género, argumentando que: «Si bien aquello que consideramos "femenino" o "masculino" se basa en una diferencia biológica sexual, estas son nociones que vamos construyendo día a día, en nuestras interacciones [...]» (p. 16). Aunque se hace referencia al presupuesto biológico que diferencia a los sujetos, inmediatamente se desdeña al señalar que esta identidad se construye día a día. No se puede negar que los patrones culturales influyen en la caracterización de cada género (femenino o masculino), pero estos no pueden edificarse al margen del sexo. Ahora bien, el currículo incide en la construcción de la identidad del niño, y eleva este concepto a la categoría de competencia de aprendizaje.

Utiliza los términos identidad sexual e identidad de género de manera confusa. Los hace independientes, cuando deberían corresponderse. Hace referencia a los derechos sexuales y reproductivos, sin embargo no los define. Como se ha mencionado, la teoría de género comprende que los derechos sexuales y reproductivos postulan como principio la libertad sexual, el ejercicio de la sexualidad sin consecuencias, el uso indiscriminado de métodos anticonceptivos, entre ellos, el aborto.

Plantea el ejercicio de la sexualidad desconociendo su trascendencia para el desarrollo de la persona e introducen términos que inicialmente no se encuentran planteados como metas para el desarrollo de capacidades de aprendizaje. Estos son «placer»y «diversidad». Cabe destacar que, en el proceso de elaboración de esta norma, no se contó con la participación de los padres de familia, pese a que la Constitución Política señala que los padres tienen el derecho de intervenir en el proceso educativo (art. 13.ํ) y a través de la sociedad, a participar en la formulación de las políticas educativas (arts. 7.ํy 22.ํ de la Ley General de Educación: Ley 28044).

El currículo sostiene que el estudiante ha cumplido de manera destacada con el estándar de aprendizaje cuando «vive su sexualidad de manera responsable y placentera» (p. 31). En este sentido, entonces, (I) se asume que los niños menores de 
18 años — que son los destinatarios del currículo - tienen ya actividad sexual; (II) lejos de promover una cultura de castidad y de respeto por uno mismo desde una perspectiva integral de la sexualidad, se desvincula la sexualidad con su sentido transcendente y queda reducida a un mero ámbito de placer.

Es necesario advertir que en este trabajo no se ha hecho referencia a las políticas y normas con enfoque de género que aún no son parte del ordenamiento jurídico, pues se encuentran en calidad de proyectos en el Poder Legislativo. Estos proyectos - como es de esperarse - proponen la regulación de la unión civil y matrimonio igualitario, la tipificación de los crímenes de odio, la despenalización del aborto para los casos de violación, malformaciones congénitas incompatibles con la vida e inseminación artificial no consentida, la regulación de la maternidad subrogada (o vientre de alquiler), la regulación de las técnicas de reproducción asistida, la aprobación de la ley de identidad de género, entre otras.

\section{Conclusiones}

La familia es una institución natural y ámbito primario de desarrollo integral del ser humano, cuyas funciones no pueden ser sustituidas por ninguna otra institución (al menos en su integralidad) y por ello se convierte en un elemento importante para el desarrollo adecuado del tejido social. La identidad de la familia es el fundamento de la obligación de protección del Estado, que debería materializarse a través de la generación de políticas públicas dirigidas a la problemática familiar.

Actualmente, las políticas públicas relacionadas con la familia se han desarrollado bajo el enfoque de género. Esto supone una contradicción, debido a que este tiene un fuerte contenido ideológico que parte del hecho de afirmar que las relaciones asimétricas (de abuso, explotación y desigualdad) que existen entre hombres y mujeres tienen en la familia su principal aliado, en la medida que desde ella se formulan y refuerzan los estereotipos que afectan a la mujer.

Si bien existen distintas formas de entender el enfoque de género (puede ser desde el sistema sexo-género o desde las teorías de las relaciones de género), se pueden identificar dos aspectos: el primero, considerar que existen determinados «roles asignados» a las mujeres (maternidad, matrimonio o cuidado) que, al ser producto del patriarcado (esto es, el dominio de los hombres), las ponen en desventaja, y el segundo, que la única manera de que la mujer se libere es adquiriendo plenos derechos sobre su sexualidad y sobre su cuerpo (favoreciendo el aborto).

A partir de sus postulados, podemos señalar que una política dirigida a la familia, que tenga incorporado el enfoque de género, no podrá contribuir con el fortale- 
cimiento de ella o con la revalorización de la maternidad, porque - por su contenido- es incompatible con estas realidades. Asimismo, no podría aportar a la armonía y a la convivencia pacífica de los peruanos, pues, en su afán de «visibilizar» a la mujer, deja en un segundo plano a los niños y a los varones: todo se construye en clave de conflicto y de culpas atribuidas, donde la mujer es una víctima del varón y del «patriarcado».

Ante la insuficiencia del enfoque de género para abordar de manera adecuada la problemática de la familia, se pone en evidencia la necesidad de contar con una propuesta - dentro de las políticas públicas- que revalorice el papel de la familia en la sociedad; poniendo énfasis en la persona humana como sujeto familiar y cuyo desarrollo precisamente se inicia por y desde la familia, lo que la convierte en la institución natural más importante de la sociedad y, en consecuencia, acreedora de la protección de esta y del Estado.

\section{Referencias}

AA. VV. (2007). Principios de Yogyakarta. Principios sobre la aplicación de la legislación internacional de derechos humanos en relación con la orientación sexual y la identidad de género. Recuperado el 21 de noviembre de 2019 de: http://yogyakartaprinciples.org/wp-content/uploads/2016/o8/principles_ sp.pdf

Aguilar, T. (2008). El sistema sexo género en los movimientos feministas. doi: 10.4000/amnis. 537

Aparisi, Á. (2002). Las nuevas tecnologías reproductivas: Presupuestos ideológicos y consecuencias para la mujer. En Aparisi, Á., \& Ballesteros, J. Por un feminismo de la complementariedad. Pamplona: EUNSA.

Aparisi, Á. (2009). Ideología de género: De la naturaleza a la cultura. Persona y Derecho. Revista de fundamentación de las Instituciones Jurídicas y de Derechos Humanos, (61), 169-193. Recuperado el 22 de noviembre de 2019 de: http:// dadun.unav.edu/bitstream/10171/17718/1/51917935.pdf

Balmaceda, J. (2017). Crímenes de odio: Comentarios al D. Leg. N. 1323 que fortalece la lucha contra el feminicidio, la violencia familiar y la violencia de género. Gaceta Penal y Procesal Penal, 5(93), 38-54.

Butler, J. (2007). El género en disputa: El feminismo y la subversión de la identidad (trad. Muñoz, M. A.). Barcelona: Paidós.

Conesa, F. (2004). La urgencia de la pastoral familiar: Los nuevos signos de los tiempos. Scripta Fulgentina, 14(27-28), 33-56.

Consejo Pontificio para la Familia. (2004). Lexicón: Términos ambiguos y discutidos sobre familia, vida y cuestiones éticas. Madrid: Ediciones Palabra. 
Corte Interamericana de Derechos Humanos. (2012). Caso Artavia Murillo y otros («fecundación in vitro») vs. Costa Rica. Recuperado el 21 de noviembre de 2019 de: http://www.corteidh.or.cr/docs/casos/articulos/seriec_257_esp.pdf

D'Agostino, F. (2002). Elementos para una Filosofía de la Familia. Madrid: Rialp.

Elósegui, M. (2005). ¿En qué, por qué y para qué somos diferentes varones y mujeres? Thémata: Revista de filosofía, 35, 125-136. Recuperado el 15 de mayo de 2019 de: http://institucional.us.es/revistas/themata/35/12\%2oelosegui.pdf

Facio, A., \& Fries L. (Eds.). (1999). Género y derecho. Santiago de Chile: LOM.

Fernández, L. (2008). La cuestión feminista hoy. Valencia: Instituto Social León XIII, Fundación Pablo VI.

Firestone, Sh. (1976). La dialéctica del sexo. Barcelona: Kairós.

Gil, W., \& Vallejo, J. (2013). Lo masculino y lo femenino: Diferentes, iguales y recíprocos. Revista Berit Internacional, 11 (14).

Instituto Nacional de Defensa de la Competencia y la Propiedad Intelectual. (2017). Resolución N.․ 1884-2017/SPC-Indecopi.

Instituto Nacional de Estadística e Informática. (2016). Perú: Encuesta demográfica y de salud familiar 2016. Lima: INEI.

Instituto Nacional de Estadística e Informática. (2018). Perú: Indicadores de violencia familiar y sexual 2009-2018. Recuperado el 17 de junio de 2019 de: https://www.inei.gob.pe/media/MenuRecursivo/publicaciones_digitales/ Est/Lib1584/libro.pdf

Melina, L. (2015). La familia, bien antropológico fundamental. La familia como sujeto social en el mundo económico. El don, fundamento del interés. En Kampowski, S., \& Gallazzi, G. (Comp.). Familia y desarrollo sostenible. Arequipa: Universidad Católica San Pablo.

Mendoza, W., \& Subiría, G. (2013). El embarazo adolescente en el Perú: Situación actual e implicancias para las políticas públicas. Revista Peruana de Medicina Experimental y Salud Pública, 30(3), 471-479.

Ministerio de la Mujer y Poblaciones Vulnerables. (2012). Plan Nacional de Acción por la Infancia y la Adolescencia 2012-2021. PNAIA 2021. Lima: MIMP.

Ministerio de la Mujer y Poblaciones Vulnerables. (2014). Si no me nombras, no existo: Promoviendo el uso del lenguaje inclusivo en las entidades públicas. Lima: MIMP.

Ministerio de la Mujer y Poblaciones Vulnerables. (2016). Plan Nacional Contra la Violencia de Género 2016-2021. Lima: MIMP.

Ministerio de la Mujer y Poblaciones Vulnerables. (2016a). Plan Nacional de Fortalecimiento a las Familias 2016-2021. Recuperado el 15 de mayo de 2019 de: http://www.mimp.gob.pe/files/planes/PLANFAM-2016-2021.pdf 
Ministerio de la Mujer y Poblaciones Vulnerables. (2017). Primer informe anual del Plan Nacional de Fortalecimiento a las Familias 2016-2021. Recuperado el 15 de mayo de 2019 de: https://observatoriodelasfamilias.mimp.gob.pe/archivos/planfam/informeplanfam2017.pdf

Ministerio de la Mujer y Poblaciones Vulnerables. (2019). Política Nacional de Igualdad de Género. Recuperado el 16 de junio de 2019 de: http://elperuano. pe/NormasElperuano/2019/04/04/1757065-1/1757065-1.htm

Montaño, S. (2005). ¿Políticas de familia versus políticas de género? En Arriaga, I. (Ed.). Políticas hacia las familias, protección e inclusión sociales (pp. 97102). Santiago de Chile: CEPAL-UNFPA. Recuperado el 14 de junio de 2019 de: https://repositorio.cepal.org/bitstream/handle/11362/6805/So5683_es.pd$\mathrm{f}$ ? sequence $=1$ \&isAllowed $=\mathrm{y}$

Mujeres Radicales. (2001). El manifiesto de las Mujeres Radicales. Estados Unidos: Radical Women Publications.

Pacora-Portella, P. (2014). Aborto terapéutico: ¿Realmente existe? Acta Médica Peruana, 31(4), 234-239.

Puyana, Y. (2007). El familismo: Una crítica desde la perspectiva de género y el feminismo. En Puyana, Y., \& Ramírez, M. H. (Eds). Familias, cambios y estrategias (pp. 263-278). Bogotá: Universidad Nacional de Colombia. Recuperado el 12 de junio de 2019 de: http://bdigital.unal.edu.co/1363/19/18CAPI17.pdf

Stolke, V. (2004). La mujer es puro cuento: La cultura del género. Revista Estudios Feministas, 12(2). Recuperado el 23 de noviembre de 2019 de: https://www. redalyc.org/articulo.oa?id=381/38112205

Torío, S., Peña, J., \& Rodríguez, M. (2008). Estilos educativos parentales: Revisión bibliográfica y reformulación teórica. Teoría educativa, (20), 151-178.

Tribunal Constitucional. (2015). STC N.o 06040-2015-PA/TC.

UNICEF. (2005). Guía para la democratización de las familias. Recuperado el o de junio de 2019 de: https://www.unicef.org/ecuador/Democratizacion_de_las_ familias_Argentina.pdf

UNICEF. (2013). Superando el adultocentrismo, Chile. Recuperado el 10 de junio de 2019 de: http://unicef.cl/web/wp-content/uploads/2012/12/UNICEF-04SuperandoelAdultocentrismo.pdf

Vivanco, A. (2006). La aplicación del concepto de autopoiesis en los estudios de género. Acta Bioethica, 12(2), 151-164. 